\title{
CRITERIOS DE ASEGURAMIENTO DE CALIDAD PARA RESULTADOS DE ENSAYO EN LADETERMINACIÓN DE BARIO POR ESPECTROMETRÍA DE ABSORCIÓN ATÓMICA EN AGUA RESIDUAL MEDIANTE CARTAS DE CONTROL DE SHEWHART QUALITY ASSURANCE CRITERIA FOR TEST RESULTS ON THE DETERMINATION OF BARIUM BY ATOMIC ABSORPTION SPECTROMETRY IN WASTEWATER USING SHEWHART CONTROL CHARTS
}

\author{
José W. Camero Jiménez ${ }^{1}$, Víctor A. Huamaní León ${ }^{2}$
}

\begin{abstract}
RESUMEN
El presente trabajo define como criterio de aseguramiento de calidad de resultados, para la determinación de Bario por el método Standard Methods 3111 D - Direct Nitrous Oxide Acetylene Flame Method por espectrometría de absorción atómica, los límites de control estimados de cartas de control individuales Shewhart para los parámetros de veracidad y precisión.El estudio se realizó enagua residual.
\end{abstract}

Palabras clave: aseguramiento de calidad, bario, carta control Shewhart, agua residual,

\begin{abstract}
This paper defines the criterion of quality assurance results for the determination of barium by the method Standard Methods 3111 D - Direct Nitrous Oxide - Acetylene Flame Method by atomic absorption spectrometry, estimated for individuals Shewhart control limits for accuracy and precision parameters. The study was conducted for wastewater.
\end{abstract}

Keywords: Assurance quality, barium, Shewhartcontrol chart, wastewater

\section{INTRODUCCIÓN}

En todo proceso de medición los resultados de ensayo contiene cierto grado de variabilidad a consecuencia de diversos factores, esto implica, que el proceso de medición no obtenga resultados de ensayo con valor constante. El objetivo del control estadístico de procesos (CEP) es establecer y mantener un proceso a un nivel aceptable y estable, asegurándose la conformidad de los resultados; siendo la carta control una de las herramientas a utilizar.
En los gráficos de control encontramos dos clases de variabilidad, la primera clase es la aleatoria debida a "causas aleatorias" también se le conoce como "causas fortuitas"; la segunda clase de variabilidad representa un cambio real en el proceso, los cuales no son parte inherente del proceso, también se les conoce como "causas asignables", esta pueden ser atribuidas a: falta dehomogeneidad en la muestra (materia prima), personal no autorizado (mano de obra), equipos sin verificar o sin calibrar (máquinas), métodos de ensayo sin

\footnotetext{
${ }^{1}$ Lic. en Estadística, Catedrático Escuela Profesional de Ingeniería Estadística (EPIES)-UNI, en Post Grado de la UNALM y consultor en Sistemas de Gestión de Calidad de laboratorios de ensayo, ${ }^{2}$ Bachiller en Química, analista químico en laboratorio ambiental de EQUAS S.A.
} 
Criterios de aseguramiento de calidad para resultados de ensayo en la determinación de

implementar adecuadamente (métodos)o condiciones de trabajo inadecuadas., etc.

Cuando un proceso opera únicamente bajo "causas aleatorias" se dice que está bajo control estadístico; sin embargo si opera bajo las "causas asignables" se dice que se encuentra fuera de control estadístico.

Por lo tanto, se define una carta control como un gráfico sobre el cual algunas mediciones estadísticas de una serie de muestras son graficadas en un orden particular para dirigir el proceso con respecto a aquella medida para controlar y reducir la variación [1].

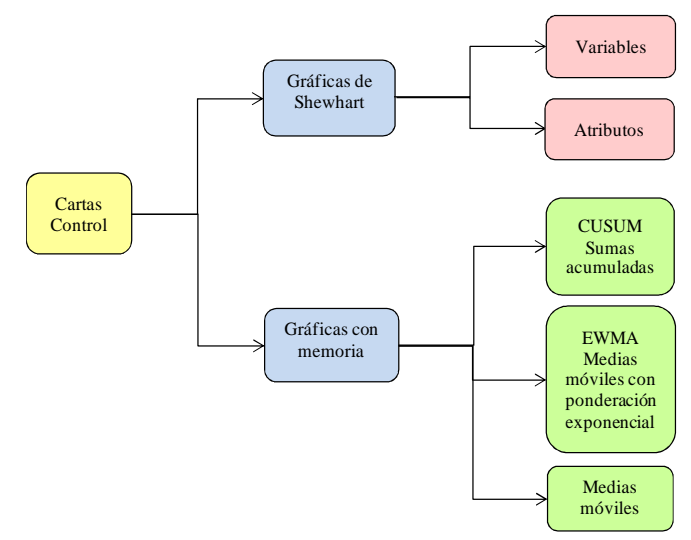

Figura 1.Clasificación de cartas control.

Existen dos tipos de cartas de control de Shewhart:

- Cuando el resultado puede medirse y expresarse como un número en una escala de medición continua, se denominan cartas control por variables (valores continuos).

- Cuando no se puede medir o dar una escala cuantitativa, en este caso se clasifica como producto conforme o disconforme, se denominan cartas control por atributos (valores discretos).
Tabla 1. Tipos de carta control de Shewhart

\begin{tabular}{|c|l|}
\hline Tipo & \multicolumn{1}{|c|}{ Nombre } \\
\hline \multirow{4}{*}{ Variables } & Gráfica $\bar{x}-R$ (valor promedio y rango ) \\
\cline { 2 - 3 } & Gráfica $x$ \\
\hline \multirow{4}{*}{ Atributos } & Gráfica $p n$ (número de unidades defectuosas) \\
\cline { 2 - 3 } & Gráfica $p \quad$ (Fracción de unidades defectuosas) \\
\cline { 2 - 3 } & Gráfica $c \quad$ (Número de defectos) \\
\cline { 2 - 3 } & Gráfica $u \quad$ (Número de defectos por unidad) \\
\hline
\end{tabular}

Para nuestro caso en estudio el tipo de carta que utilizaremos son los gráficos de control de Shewhart para datos individuales, debido a que el tamaño de muestra usado para monitorear el proceso es $n=1$.

En el laboratorio de ensayo, un factor importante para la construcción de las cartas control es la muestra control, la cual nos ayudará a monitorear todo el proceso analítico. Esta muestra control debe ser estable durante el tiempo; pudiendo ser: una sustancia patrón, muestra sintética fortificada o adicionada, material de referencia, material de referencia certificado, muestra real [2].

Se determinara tres gráficas de control para la técnica de Bario:

- Proceso: Muestra control sintética (preparado a partir de un estándar de Bario).

- Veracidad: Muestras fortificadas y expresadas como porcentaje de Recuperación.

- Precisión: Duplicados del porcentaje de Recuperación y expresados como \%RSD (desviación estándar relativa de los duplicados). 


\section{FUNDAMENTO DE LAS CARTAS CONTROL DE SHEWHART PARA INDIVIDUOS}

Para controlar un proceso siguiendo la evolución de una variable, es importante poder controlar un parámetro de posición central y un parámetro de dispersión.

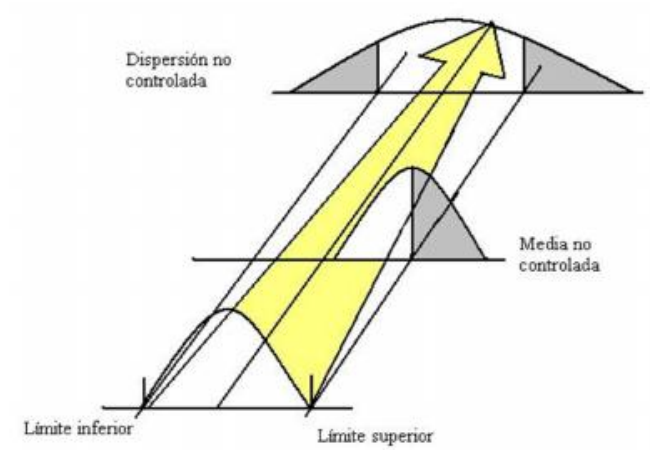

Figura 2.Tendencia central y dispersión no controlada en una carta control.

Entonces, sea "x" la variable de interés la cual sigue una distribución normal, en los cuales el valor esperado y la varianza vienen dados por:

$E(x)=\mu$

$\operatorname{Var}(\mathrm{x})=\sigma^{2}$

Los cuales se expresa como intervalos de confianza, obteniéndose:

Límite de control superior

$\mathrm{UCL}=\mu+\mathrm{K} \sigma$

Límite de control inferior

$\mathrm{LCL}=\mu-\mathrm{K} \sigma$

Donde $\mathrm{K}$ es el valor asociado al nivel de confianza de $99,7 \%$ de una distribución normal, tomando un valor aproximado de $\mathrm{K}=3$.

El problema es que $\sigma$ es un estimador sesgado; por lo tanto debe usarse otro estimador de la dispersión [3], en este caso:
$\hat{\xi})=\frac{\bar{R}}{d_{2}}$

Dondee $\hat{\S}$ un estimador insesgado de $\sigma$;

$\bar{R}=\frac{\sum_{i=1}^{K} R_{i}}{K}$

Q el rango promedio; $\mathrm{R}_{\mathrm{i}}$ es el rango en cada subgrupo; $\mathrm{K}$ es el número de muestras, generalmente entre 20 a 25 [4],[5]; $d_{2}$ es una constante igual:

$\mathrm{d}_{2}=1.128$

Reemplazando (5) en (3) y (4):

Limites $=\mu \pm 3 \frac{\bar{R}}{d_{2}}$

Para cartas control con datos individuales se reemplaza $R$ por el rango móvil promedio $\overline{M R}$

$$
\overline{M R}=\frac{\sum_{i=2}^{K} M R_{i}}{(K-1)}
$$

Donde

$$
M R_{i}=\left|x_{i}-x_{i-1}\right|
$$

$\mathrm{MR}_{\mathrm{i}}$ es el rango móvil de extensión dos $(i=2,3, \ldots, K$.)

Por lo tanto, los límites para la tendencia central en la carta control para individuos serian reemplazando $\mu$ por la media muestral y la ecuación (9) en (8), obteniéndose:

Límite de control superior

$U C L=\bar{x}+3 \frac{M R}{d_{2}}$

Límite de control inferior

$$
L C L=\bar{x}-3 \frac{\overline{M R}}{d_{2}}
$$


Criterios de aseguramiento de calidad para resultados de ensayo en la determinación de

Los límites para la variabilidad en función al rango móvil serian parecidos al de la tendencia central, por lo tanto:

Límite de control superior de la carta $\mathrm{R}$

$$
U C L=D_{4} \overline{M R}
$$

Límite de control inferior de la carta $\mathrm{R}$

$$
L C L=D_{3} \overline{M R}
$$

Donde $\mathrm{D}_{3}=0$ y $\mathrm{D}_{4}=3.267$; las cuales son constantes que dependen del tamaño de subgrupo.

El método analítico está bajo control si los resultados obtenidos con este método siguen una distribución normal; entonces [4]:

- Aproximadamente el $67 \%$ de los resultados han de encontrarse dentro del intervalo de control $\pm 1 \mathrm{~s}$. (siendo $\mathrm{s}$ el estadístico de la dispersión).

- aproximadamente el 95\% de los resultados han de encontrarse dentro del intervalo de control $\pm 2 \mathrm{~s}$.

- aproximadamente el $99 \%$ de los resultados han de encontrarse dentro del intervalo de control $\pm 3 \mathrm{~s}$

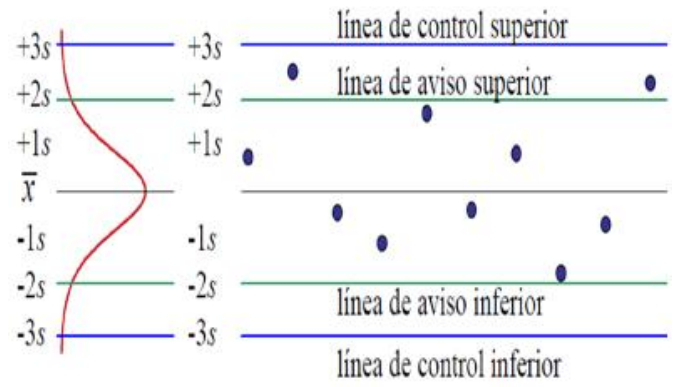

Figura 3.Límites de control y de aviso en una carta control.

\section{CAUSAS ASIGNABLES EN LAS CARTAS CONTROL}

Para una mejor visualización, el grafico se divide equitativamente en seis zonas, teniendo en cada zona un ancho de $1 \sigma$; las cuales se rotulan como A, B, C, C, B, A. Teniendo 8 causas asignables [4]:

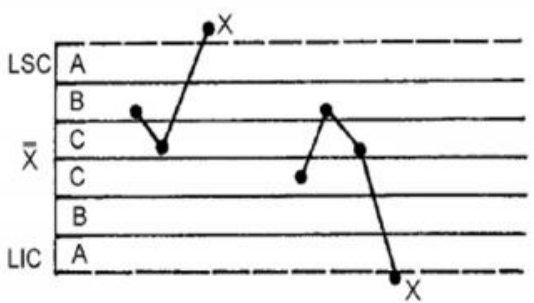

Figura 4.Caso 1: Un punto más allá de la zona $A$.

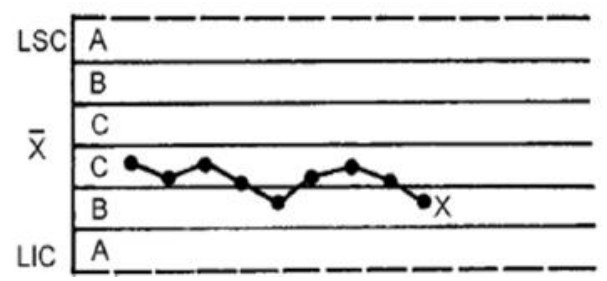

Figura 5. Caso 2: Nueve puntos en una fila en la zona $C$ o más allá en un lado de la línea central.

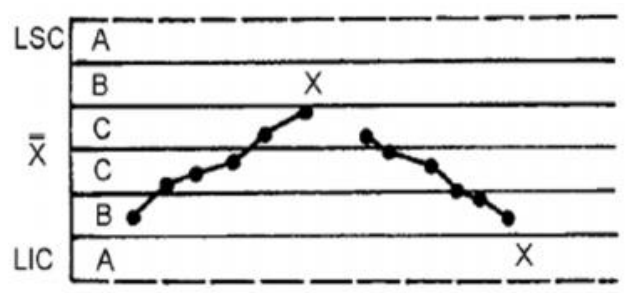

Figura 6. Caso 3: Seis puntos en una fila que aumenta o disminuye en forma constante.

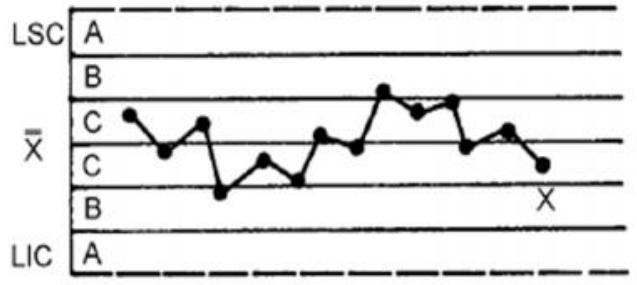

Figura 7. Caso 4: Catorce puntos en una fila alternante hacia arriba y hacia abajo. 


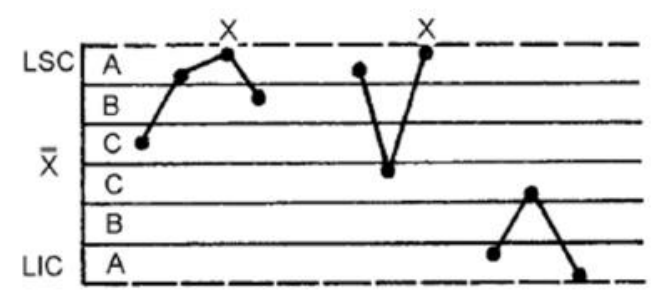

Figura 8. Caso 5: Dos de tres puntos en una fila en la zona A o más allá.

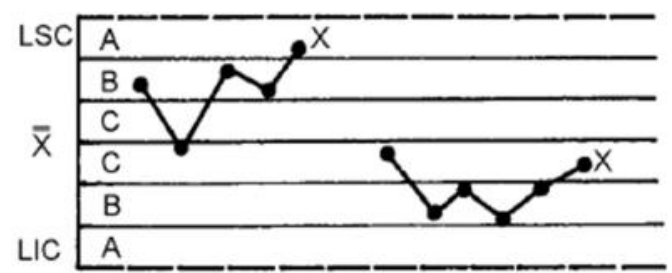

Figura 9. Caso 6: Cuatro de cinco puntos en una fila en la zona $B$ o más allá.

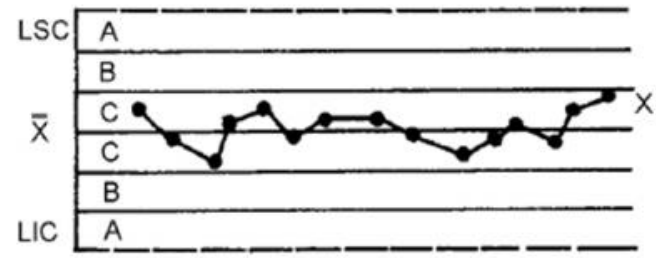

Figura 10.Caso 7: Quince puntos en una fila en la zona $C$ por encima o por debajo de la línea central.

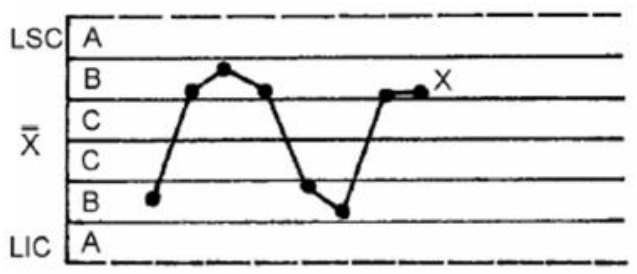

Figura 11. Caso 8: Ocho puntos en una fila en ambos lados de la línea central con ninguno en la zona $C$.

\section{DESARROLLO DE LAS CARTAS CONTROL}

Se evaluó 20 muestras durante el tiempo de análisis de rutina de la técnica de bario por espectrometría de absorción atómica para la matriz de agua residual.

\section{Carta control para las muestras control de bario.}

Se evidencio que siguen una distribución normal para los datos.

La muestra control para el método de bario se expresa como un rendimiento, quiere decir:

$$
\% R=\frac{\text { Valor_experimental }}{\text { Valor_teórico }} x 100 \quad \text { ) }
$$

Donde el valor experimental es el resultado del sintético a través de todo el proceso de digestión para el bario $(\mathrm{mg} / \mathrm{L})$; valor teórico es el valor esperado del preparado sintético (mg/L).

Tabla 2. Resultados para el cálculo de los límites de control.

\begin{tabular}{|c|c|}
\hline$\overline{M R}$ & 2.955 \\
\hline $\bar{x}$ & 98.11 \\
\hline $\mathbf{K}$ & 20 \\
\hline $\mathbf{d}_{\mathbf{2}}$ & 1.128 \\
\hline $\mathbf{D}_{\mathbf{3}}$ & 0 \\
\hline $\mathbf{D}_{\mathbf{4}}$ & 3.267 \\
\hline
\end{tabular}

Aplicando las ecuaciones (11), (12), (13) y (14) se obtuvo lo siguiente:

Límites para $\mathrm{x}$ :

$\mathrm{UCL}=105.97 \%$

$\mathrm{LCL}=90.25 \%$

Gráficamente los resultados son los siguientes: 
Criterios de aseguramiento de calidad para resultados de ensayo en la determinación de

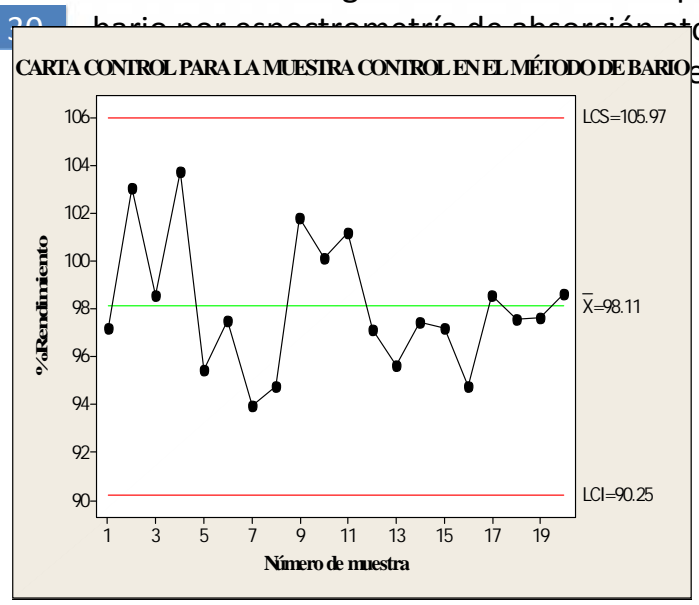

Figura 12.Grafica de X para la muestra control de bario.

\section{Carta Control para el sesgo expresado como muestras fortificadas para el Bario.}

Se evidenció que siguen una distribución normal para los datos.

Las muestras fortificadas se hicieron agregando una concentración conocida a las muestras y se calculó su recuperación, utilizando la siguiente ecuación:

$\% \operatorname{Re}=\left(\frac{C^{\prime}-C_{0}}{C_{A d}}\right) \times 100 \%$

$\%$ Re es el porcentaje de recuperación;

$C^{\prime}$ es la concentración fortificada (mg/L);

$\mathrm{C}_{0}$ es la concentración nativa $(\mathrm{mg} / \mathrm{L})$;

$\mathrm{C}_{\mathrm{Ad}}$ es la concentración adicionada (mg/L).

Tabla 3. Resultados para el cálculo de los límites de control.

\begin{tabular}{|c|c|}
\hline$\overline{M R}$ & 3.741 \\
\hline $\bar{x}$ & 98.75 \\
\hline $\mathbf{K}$ & 20 \\
\hline $\mathbf{d}_{\mathbf{2}}$ & 1.128 \\
\hline
\end{tabular}

Límites para $\mathrm{x}$ :

$\mathrm{UCL}=108.70 \%$ y LCL $=88.80 \%$

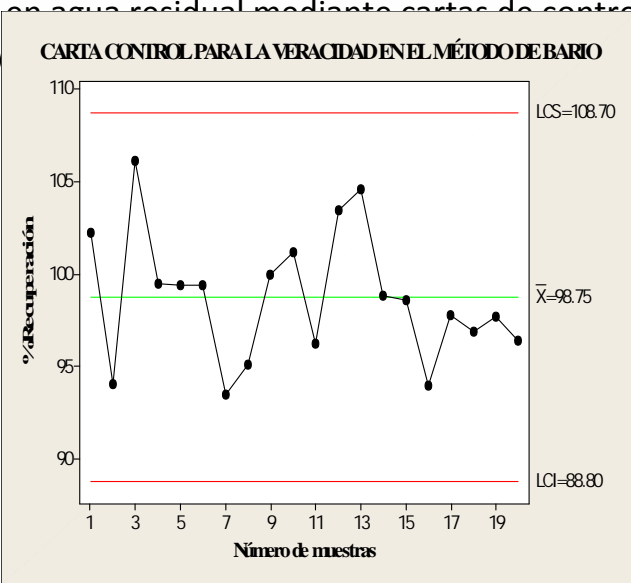

Figura 13.Grafica de X para la veracidad del método durante el tiempo.

Según [8] el rango de aceptación en la \%Recuperación es de $80-110 \%$, por lo tanto el rango estimado en la carta control es veraz.

\section{Carta Control para la precisión expresado \% RSD para el Bario.}

Los duplicados expresados como \%RSD, se calculó utilizando la ecuación [6], [7]:

$R S D_{r}=\sqrt{\frac{\sum_{i=1}^{n} d_{i}{ }^{2}}{2 n}}$

$d_{i}=\frac{\left(a_{i}-b_{i}\right)}{-} \times 100$

Donde:

$a_{i} \quad \mathrm{y} \quad b_{i}$ son los resultados de las determinaciones duplicadas $\left(\mathrm{mg} / \mathrm{L} \bar{\alpha}_{j} ; \mathrm{es}\right.$ el valor medio de cada duplicado $(\mathrm{mg} / \mathrm{L}) ; n$ es el número de determinaciones duplicadas.

Tabla 4. Resultados para el cálculo de los límites de control.

\begin{tabular}{|c|c|}
\hline$\overline{M R}$ & 0.525 \\
\hline $\mathbf{K}$ & 20 \\
\hline $\mathbf{D}_{\mathbf{3}}$ & 0 \\
\hline $\mathbf{D}_{\mathbf{4}}$ & 3.267 \\
\hline
\end{tabular}

Límites para el Rango móvil:

$\mathrm{UCL}=1.717 \%$ y LCL $=0.000 \%$ 


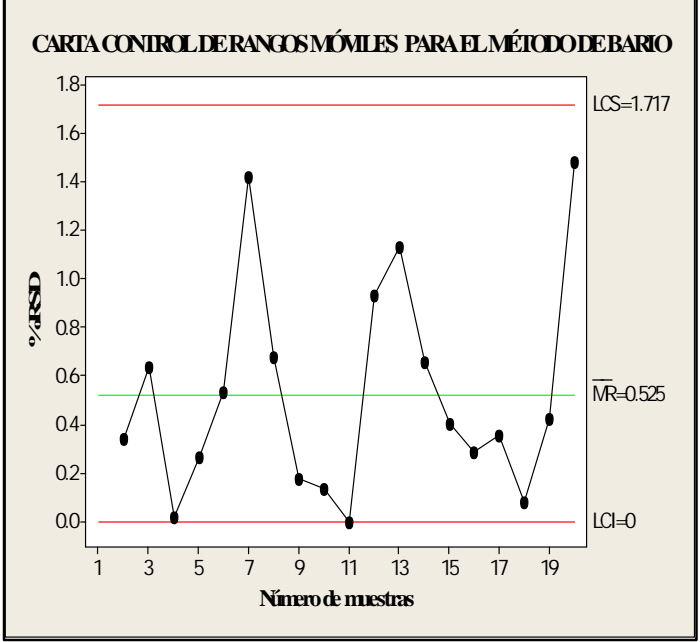

Figura 14.Gráfica de rangos móviles para la precisión para el método de bario.

Un rango aceptado para los duplicados según [7] es que la diferencia sea menor al valor de 5\%; por lo cual los duplicados expresados como \%RSD son precisos.

\section{CONCLUSIONES}

- Las tres cartas control elaboradas para la determinación de bario por espectrometría de absorción atómica para la matriz de agua residual, están libres de puntos fuera de los límites de control, no se evidencia rachas con causas asignables, tampoco se evidencia tendencias ni periodicidad, por lo tanto la variabilidad reflejada es solo a causa de efectos aleatorios.

- La recuperación para muestras control se establece entre $90.25 \%$ a $105.97 \%$, como criterio de aseguramiento de calidad de resultados a futuro para esta técnica analítica en la matriz de agua residual.

- La recuperación para muestras fortificas (veracidad) se establece entre $88.8 \%$ a $108.7 \%$, como criterio de aseguramiento de calidad de resultados a futuro para esta técnica analítica en la matriz de agua residual.
- El RSD máximo permitido para duplicados será $1.717 \%$, como criterio de aseguramiento de calidad de resultados a futuro para esta técnica analítica en la matriz de agua residual.

- Los límites establecidos en la elaboración de las cartas control son dinámicos, debido a esta propiedad deben renovarse cada cierto tiempo programado o cuando se vea afectado un factor determinante en el análisis del método.

- En el caso de que se halle en los análisis de rutina un dato anómalo, debe investigarse la razón de esta y corregirlo, para poder así mantener en control estadístico el método analítico.

\section{REFERENCIAS}

1. INDECOPI, Norma técnica peruana, NTP-ISO 7870:2008. "Gráficos de control. parte 1: directrices generales". Primera edición.

2. Riu, Jordi. "Gráficos de control de Shewhart".Universitat Rovira i Virgili.

3. Montgomery, Douglas. "Control estadístico de la calidad”. Tercera edición, Editorial LimusaWiley.

4. Norma técnica colombiana, NTCISO 8258. "Gráficos de control de Shewhart", ICONTEC.

5. Vachette, Jean-Luc. "Mejora continua de la calidad. Control estadístico de proceso SPC" Ediciones CEAC S.A. Barcelona, 1992. 
Criterios de aseguramiento de calidad para resultados de ensayo en la determinación de

6. Procedimiento NMKL $\mathrm{N}^{\circ} 5$, "Estimación y expresión de la incertidumbre de la medición en análisis químico", 2005.

7. APHA, AWWA, WEF. "Standard Methods for the Examination of Water and Wastewater", January 2012, 22 St Ed. Washington.

8. AOAC, AssocOff Anal Chem, 1993; Huber, 1999. En: AEFI, Validación de métodos analíticos, Cataluña, 2001, pp. 84.

Correspondencia: jcameroj@uni.edu.pe

Recepción de originales: enero 2013

Aceptación de originales: abril 2013 